\title{
Análisis económico de predios lecheros del sur de Chile
}

\author{
Economic analysis of dairy farms in southern Chile \\ J. Lerdon*1, I. Herrera ${ }^{1}$, V. Moreira ${ }^{1}$, B. Carrillo ${ }^{2}$
}

\section{RESUMEN}

Se presenta una caracterización productiva, social y económica de 13 predios productores de leche, de la Región de Los Lagos (sur de Chile). El período de estudio contempló la temporada 2012-2013. Con los datos se generó un inventario de activos y pasivos, ingresando la información al Sistema de Contabilidad de Gestión Agrícola (CONGA), del Instituto de Economía Agraria de la Universidad Austral de Chile. Con este se realizó un análisis económico y financiero, y se determinaron las principales variables productivas. A partir del análisis se obtuvieron valores de solvencia, liquidez, productividad económica y rentabilidad. También se determinaron variables sociales evaluando escolaridad, sexo, estado civil, número de hijos y edad de los agricultores. Los resultados indican que la edad promedio es inferior a lo encontrado por otros autores. Al compararla con los beneficios netos, a menor edad se presenta un mejor indicador. El grado de escolaridad es nivel técnico para la mayoría de los productores del grupo, teniendo estos estudios agrarios. El beneficio neto total fue positivo en todos los predios del estudio; siendo los costos más elevados los de la alimentación, seguido por la reposición y la fertilización. Las rentabilidades promedio fueron superiores a lo encontrado por otros autores. La mayor correlación positiva se registró entre la edad y el margen directo por cabeza y entre la edad y el margen directo por hectárea. En el caso de estos mismos productores, el grado de endeudamiento y el nivel de escolaridad se correlacionaron negativamente, observándose esta misma situación al correlacionar el beneficio neto por hectárea y el nivel de escolaridad en este rubro de lechería.

Palabras clave: estudio de casos, análisis económico, producción de leche.

\section{ABSTRACT}

A productive, social and economic characterization of 13 milk farms, located in the Region of Los Lagos, in southern Chile is presented. The period of study involved the 2012-2013 season. An inventory of theirs assets and liabilities was generated based on the data provided by the farmers, the information was uploaded onto the management software of agricultural accounting (CONGA) designed by the Institute of Agricultural Economics at the University Austral of Chile. A detailed economic and financial analysis was obtained, and the major production variables were determined. The economic-financial analysis considered the solvency, liquidity, economic productivity and profitability. Meanwhile, some variables were determined by interviews that evaluated education, sex, age, and family size. The results indicate that the average age is less than the one registered by others authors. When compared to net benefits, younger age it is a better indicator. The education level is technical for most of the farmers, with many of them having agricultural studies. Net profit is positive for all farms, and the highest production costs are replacement, feed costs and fertilizer expenses. The farm profitability is higher than average profits reported by other authors. The highest positive correlation was recorded between age and net profit by cow, and between age and net profit by hectare. In the case of these same milk farmers, the level of indebtedness and education are negatively correlated, showing the same situation for the correlation of net profit by hectare and the level of education of dairy farmers.

Key words: case studies, economic and financial analysis, milk production.

\section{Introducción}

Hoy en día las empresas agropecuarias de Chile están cambiando su visión para adecuarse al nuevo estilo de gestión al que se enfrentan empresas de diferentes rubros a nivel mundial. Este desafío ha generado para la agricultura chilena un cambio en la estructura empresarial, buscándose aumentar la competitividad.

En el escenario de la agricultura mundial, el tema central de la política agroalimentaria está enfocado en la liberalización del mercado de los productos agrícolas,

\footnotetext{
1 Instituto de Economía Agraria, Universidad Austral de Chile. Casilla 567, Valdivia, Chile.

2 Instituto de Ciencia y Tecnología de los Alimentos, Universidad Austral de Chile. Casilla 567, Valdivia, Chile.

* Autor por correspondencia: jlerdon@uach.cl
}

Fecha de Recepción: 4 Abril, 2016.

Fecha de Aceptación: 21 Septiembre, 2016.

DOI: $10.4067 / \mathrm{S} 0718-34292016005000028$ 
debido a la cantidad de subsidios a la producción, exportación e ingresos de este rubro. Sin embargo, estas políticas de Estado solo se practican en algunos países más desarrollados como la Unión Europea, Estados Unidos, Canadá, Japón. Por lo que se puede ver, en países con mayor desarrollo, las bases de la agricultura tradicional están siendo reemplazadas por nuevos sistemas, estructuras y formas de enfrentar la actividad agrícola (Gordillo y Jiménez, 2004).

En Chile, el sector agrícola ha experimentado gran expansión y modernización en los últimos años, con avances en la producción, incorporación de tecnología, mejoras en la organización institucional, situación social y económica. Sin embargo, se mantienen conflictos en la pequeña agricultura, la que ha quedado atrás en adelantos tecnológicos, en comparación con los agricultores grandes (Echenique, 2004).

El sector agrícola presenta problemas y desafíos. A mediados de los ochenta, la agricultura chilena se encontraba sometida a grandes tensiones, resultado de los cambios de la economía mundial. La globalización de los mercados ha generado tratados internacionales de libre comercio, en donde Chile participa activamente (Sandoval y Gómez, 1999).

Por ello, la agricultura chilena y aún más la agricultura familiar campesina, se enfrenta con la necesidad de desarrollar ventajas competitivas, mediante un esfuerzo de investigación e innovación para poder aumentar la productividad, permitiendo a los productores alcanzar niveles de competitividad y poder incorporarse a mercados nacionales e internacionales, ya que la capacidad de reacción que desempeñen será clave para enfrentar con éxito la transformación que promueven los mercados agroalimentarios (Villalobos, 2002).

El estado debe crear mecanismos económicos y financieros que permitan a la pequeña agricultura integrarse plenamente a los mercados agropecuarios modernos (OCDE, 2009).

Según Domínguez (2000), la transformación en la actividad agrícola no necesariamente radica en readecuar rubros o técnicas productivas, sino que se centra en un cambio en la estructura de la empresa, para que los agricultores respondan mejor a las demandas de los consumidores.

Respecto al tema financiero, según el mismo autor, los pequeños productores se han visto enfrentados a una disminución en la rentabilidad. Esto ha afectado a la mayoría de los rubros, provocando caídas en sus ingresos, en especial a nivel de pequeños y medianos productores que, al no tener capacidad de reacción, han aumentado su endeudamiento, salido de la actividad y/o han buscado otras alternativas.

Los principales problemas que enfrentan los campesinos son: ausencia de tecnología apropiada para la escala de producción, administración inadecuada de su rubro e ineficiente gestión empresarial, restringidas modalidades de financiamiento, dificultad para acceder a nuevos mercados y la deficiencia de sus insumos (IICA, 2004).

Para contribuir a resolver lo planteado anteriormente se requiere de una adecuada gestión financiera, que va más allá de centrar el objetivo en soluciones, para cuando se tienen problemas financieros, sino que debe preocuparse de cubrir los riesgos involucrados en la actividad de la empresa y definir políticas de desarrollo (Lerdon, 2011).

Paralelo con las herramientas de solución, hace algunos años, surgieron los Grupos de Transferencia Tecnológica (GTT), constituidos por productores con un interés común. Estos se reúnen periódicamente en el predio de uno de los integrantes, con el fin de capacitarse y analizar sus experiencias de manejo predial, para proyectar en conjunto el desarrollo de sus empresas (Becerra, 2002). Al compartir un objetivo común, mantienen características similares, como pertenecer a la misma zona agroecológica, a un nivel socioeconómico similar y a una misma orientación productiva. Buscan mejorar su capacidad técnica, para solucionar problemas y responder al mercado.

El Estado, ha implementado medidas para mejorar la eficiencia productiva y económica en empresas agropecuarias de tamaño mediano y pequeño, financiando proyectos para acortar la brecha de competitividad de los agricultores. Un ejemplo fue el proyecto, "Programa de Difusión (PDT) para Grupos de Transferencia Tecnológica (GTT) conformados por PYMES Agropecuarias del Sur de Chile", financiado por Innova Chile y desarrollado por el Instituto de Economía Agraria de la Universidad Austral de Chile; que buscó introducir conocimientos e incorporar tecnologías de gestión. Así, se contribuyó a mejorar el proceso de planificación, control de gestión y evaluación de cada empresa y en los diferentes rubros que desarrolla (Lerdon, 2012 b).

El objetivo del presente estudio, que formó parte de este proyecto, fue elaborar un diagnóstico técnico, económico y productivo, considerando algunas variables sociales, en 13 empresas del rubro 
leche, ubicadas en la Región de Los Lagos, que en conjunto conforman el GTT denominado Real.

\section{Material y Métodos}

Los datos utilizados fueron obtenidos la temporada 2012-2013, en 13 predios dedicados al rubro leche, ubicados en la Región de Los Lagos, comunas de Fresia y Los Muermos.

Se recopilaron antecedentes productivos, económicos y financieros, durante visitas a los agricultores. Los datos fueron ingresados al Sistema de Contabilidad de Gestión Agrícola (CONGA), desarrollado en el Instituto de Economía Agraria de la Universidad Austral de Chile y disponible en www.gestiondelagro.cl. Este es un conjunto de procedimientos computacionales que permiten el registro, proceso de datos y obtención de información presupuestaria y contable, resultante de la explotación de empresas agropecuarias (Lerdon, 2012 a). Se realizaron operaciones de cierre, consistentes en determinar las diferencias de inventarios, corrección monetaria y depreciación de activos.

Como variables sociales se consideró la escolaridad, sexo, estado civil, número de hijos y edad del agricultor(a), y como variables productivas la superficie aprovechable, la producción de leche (litros promedio/vaca masa/ año), la distribución de la masa ganadera, ingresos y costos, margen directo del rubro y beneficio neto total de la explotación.

Las variables económicas y financieras se relacionan con las anteriores, adicionando la valorización de los activos de cada empresa y su endeudamiento, permitiendo por medio del sistema de gestión calcular la rentabilidad económica y financiera, indicadores de liquidez (capital de operación, necesidad en capital de operación, situación de liquidez), e indicadores de solvencia (tasa de solvencia, autonomía financiera, grado de endeudamiento).

A objeto de medir el grado de relación entre las variables los datos fueron sometidos a un análisis estadístico calculando el coeficiente de correlación de Pearson.

\section{Resultados y Discusión}

\section{Características sociodemográficas de los productores(as)}

La edad promedio es de 46 años, siendo el $54 \%$ menores de 46 , y el $46 \%$ restante se distribuyen en partes iguales en los rangos de 48 a 58 años y mayores de 58 . La menor edad relativa podría facilitar el proceso de innovación y emprendimiento. $\mathrm{Al}$ respecto, Apey et al. (2005), señalan que las explotaciones en Chile estaban mayoritariamente en manos de personas mayores de 46 años (72,5\%). Lerdon et al. (2008), en un estudio realizado en 16 predios en la zona de Valdivia, encontraron que en el rango de 54-79 años se encontraba el $38 \%$, a diferencia de lo observado en el presente estudio. Al respecto, según Lerdon (2011), el aumento de la edad podría incidir negativamente en el aumento de producción de leche, ya que en la medida que esta avanza, los productores prefieren mantener sistemas productivos tradicionales antes que optar por innovación y uso de nuevas tecnologías.

La distribución de los productores según el nivel de escolaridad muestra que el $62 \%$ posee estudios técnicos, en su mayoría agrarios; un $23 \%$ enseñanza media completa y un $15 \%$ enseñanza básica completa. El alto número de productores con estudios técnicos agrícolas, es posible relacionar con un mayor nivel de conocimientos en el área, mejorando las decisiones en sus explotaciones. Sin embargo, Lerdon et al. (2008), encontraron que un $19 \%$ de los agricultores analizados presentaba estudios técnicos completos, porcentaje inferior al encontrado en el presente estudio, debido probablemente al tipo de agricultor analizado.

Según Apey et al. (2005), existe una disminución progresiva del nivel de educación de los productores, a medida que el tamaño de las explotaciones disminuye, al igual que la disponibilidad del uso de la tecnología y el capital que poseen. Por su parte, Lerdon (2012 b), señala que a medida que aumenta la educación en las personas, mayor es el grado de aceptación de la innovación, mejorando el manejo de sus explotaciones.

Respecto al sexo, pese a que el número es similar entre hombres y mujeres, destaca la alta participación de estas últimas, ya que el $54 \%$ pertenece a este grupo; el restante $46 \%$ son hombres. Este resultado, en el que el número de participantes por grupo es casi igual, es similar al observado por Díaz (2005), quien estableció los mismos porcentajes para hombres y mujeres. Por su parte, Apey et al. (2005), establecieron que en la agricultura de subsistencia la participación de las mujeres alcanzaba un $41,7 \%$, disminuyendo a un $4,1 \%$ en la agricultura empresarial. 
Según Díaz (2005), en la mayoría de los hogares rurales los "jefes de hogar" eran hombres, y tan solo el 22\% reconocía a una mujer con este cargo. Esta información normalmente está asociada a la ausencia de pareja, siendo familias monoparentales.

Por su parte, según el censo del 2002, de la totalidad de mujeres rurales, el $51 \%$ estaban casadas, mientras que el $28 \%$ estaban solteras. En un estudio posterior, Díaz (2005), encontró que del total de la población rural femenina el $47 \%$ estaban viudas y el $23 \%$ solteras, mientras que del total de la población masculina, el $72 \%$ estaban casados y el $15 \%$ mantenía una situación de convivencia.

\section{Antecedentes productivos}

Las variables productivas analizadas fueron: superficie aprovechable, producción de leche (litros promedio/vaca masa/ año), clasificación de la masa ganadera (UA), margen directo de (crianza vaca ternero, lechería), ingresos y costos, beneficio neto total del rubro y beneficio neto total de la explotación. Las cifras económicas están expresadas en moneda de marzo del 2013.

\section{Tamaño del predio y tenencia}

En la Figura 1, que muestra la superficie utilizada, se observa que el predio de mayor tamaño tiene en total 111 ha, 50 de las que utiliza en la producción lechera. La explotación más pequeña dedica una superficie de solo 9 ha (número 12) a lechería. En promedio, los productores dedican 61 hectáreas a este rubro, cifra que corresponde a un $49 \%$ de la superficie promedio total.

Lerdon et al. (2008), en un estudio realizado en 16 predios lecheros en la zona de Valdivia, registraron un rango de 3,5 a 40,2 ha destinadas a lechería, con un porcentaje promedio de utilización de $68,4 \%$ del total, cifra superior a lo observado en este estudio.

En lo que respecta a la tenencia de la tierra solo uno de los predios es arrendado (agricultor 7). En el caso de dos agricultores (2 y 6), solo una parte de su predio mantiene esta condición. Según INE (2007), al clasificar las explotaciones agropecuarias del censo, el $62 \%$ de los terrenos eran propios, mientras que el $4 \%$ se encontraba en calidad de arriendo. Ramírez (2002), señala que los arriendos permiten al agricultor acceder a la tierra con menores recursos.

\section{Masa ganadera}

En la Figura 2 se observa que el $63 \%$ de los animales existentes en los predios lecheros son vacas en producción, mientras que el $11 \%$ vaquillas

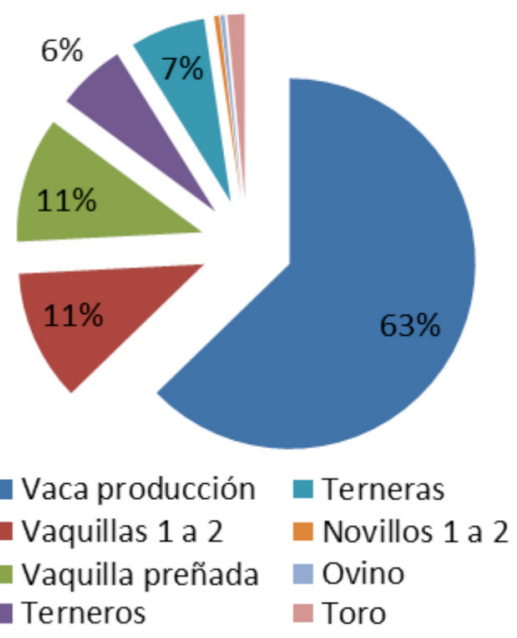

Figura 2. Masa ganadera total.

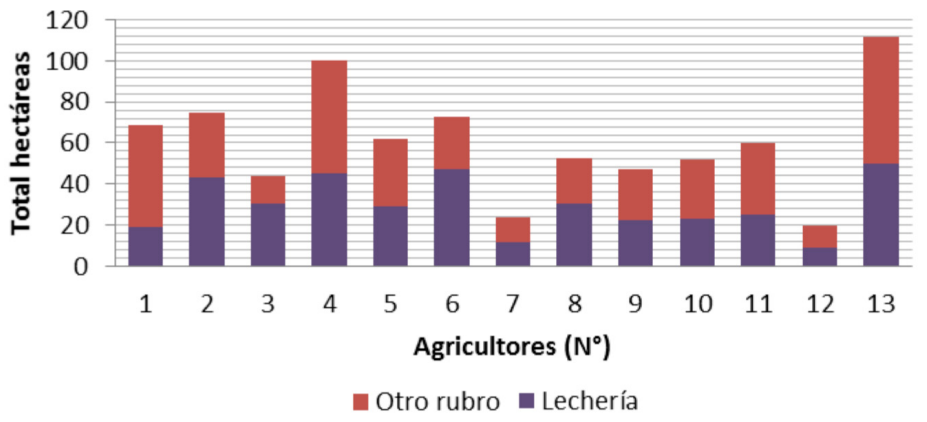

Figura 1. Superficie total destinada a la producción de leche. 
preñadas, y el mismo porcentaje vaquillas de 1 a 2 años. El expresar la masa ganadera en Unidades Animal (UA), esta varía entre 24 y 133 UA, con un promedio de 63 UA.

Lerdon y Rautenberg (2001), en un análisis de seis predios lecheros de la zona de Panguipulli, encontraron que el rebaño lechero variaba de 120 a 238 UA, valores superiores a los observados en este estudio.

\section{Producción de leche}

En la Figura 3 se puede apreciar que el predio que más leche produce por vaca masa alcanza los 6.090 litros (agricultor 6), mientras que el que produce menos (agricultor 12) alcanza 721 litros. Esta diferencia se debe probablemente a la alimentación; así en el primero, además de pradera y ensilaje, se suministra gran cantidad de concentrado en invierno; mientras que el productor 12 solo utiliza ensilaje, papas y pradera para la alimentación.

En contraste con lo registrado en el presente estudio, Lerdon et al. (2008), determinaron un promedio de 2.527 litros/vaca masa para la primera temporada y 2.619 para la segunda temporada, siendo estos promedios bastante inferiores a los encontrados en este estudio.

En Chile el promedio de producción de leche alcanzaban los 2.500 litros/ha/año, en planteles no mayores a 60 vacas (Navarro, 2006).

El agricultor que mayor número de vacas utiliza para la producción de leche (número 5) cuenta con 95 cabezas; sin embargo, no es el que produce más leche por vaca masa, pero si por hectárea (14.411 litros/ha).

Hofer (2011), señala que la zona sur es donde más se concentra la producción, alcanzando 6.000 a 12.000 litros/ha, utilizando sistemas pastoriles

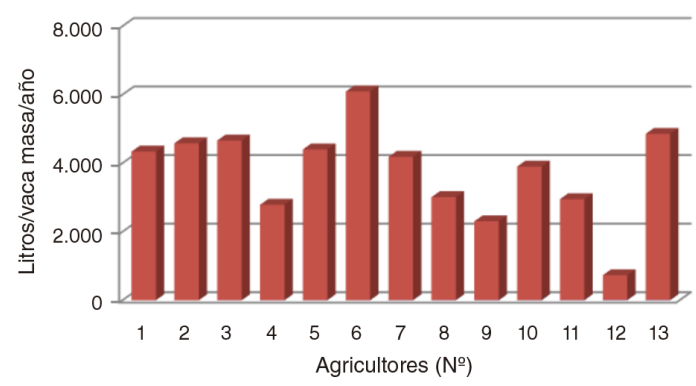

Figura 3. Producción promedio por vaca masa al año. principalmente, siendo estas cifras superiores a lo encontrado en este estudio.

Por su parte, Smith et al. (2002), señalan que en la anterior X Región se presentaban cuatro tipos de sistemas productivos lecheros, de acuerdo con aspectos técnicos y productivos, y uno de estos es el que más se asemeja a los resultados encontrados en el presente estudio. Tales características son una producción anual por vaca de 2.776 litros; concentración biestacional de pariciones o distribuidas a lo largo del año; uso de forraje conservado y concentrados para no disminuir la producción de leche en invierno.

La producción promedio por hectárea observada en este estudio fue de 6.138 litros, con un rango de 1.281 a 14.411 litros/hectárea (productores 12 y 5).

Según Balocchi (2011), en ensayos realizados en la Región de Los Lagos, específicamente en el Centro Experimental Remehue, en praderas de buena calidad se pueden obtener producciones de leche de 10.000 a 11.000 litros/ha, cifra que se incrementa por el valor nutritivo de la pradera y del potencial productivo de las vacas.

\section{Margen directo}

Corresponde al ingreso bruto del rubro. Está conformado por la venta de leche, vacas de desecho, terneros, menos los costos directos del mismo. Por el Tabla 1 se presentan los resultados del rubro lechería, observándose un margen directo total promedio de $\$ 19.770 .836$, con un rango de \$ 1.141.561 a \$ 35.608 .525 (productor 12 y 5), resultados dependientes principalmente del tamaño del rebaño (96 y 16 cabezas).

El productor con el mayor margen directo total (número 5), coincidentemente corresponde al que tiene el mayor número de cabezas (95), y mejor precio por la venta de leche (\$178/litro, en promedio); además, es un predio libre de enfermedades.

El margen por cabeza presenta un promedio de \$ 405.992; la cifra más alta (\$ 652.893) la obtuvo el productor 2. Esto se debe probablemente al ingreso por vaca, con costos mínimos de producción; mientras que el productor 12, con el margen más bajo (\$71.348), obtiene ingresos inferiores por vaca y los costos son el doble en relación al productor 2 .

En cuanto al margen directo por hectárea, se observa en la Tabla 1 que el promedio es de $\$ 664.460$, con un rango de \$ 125.229 a \$ 1.230 .930 (productor 12 y 5 ). 
Tabla 1. Margen directo.

\begin{tabular}{cccccr}
\hline Agricultores $\left(\mathrm{N}^{\circ}\right)$ & Margen directo $(\$)$ & Tamaño $(\mathrm{cab})$ & Total leche $(\mathrm{ha})$ & Margen/cab $(\$)$ & Margen/ha $(\$)$ \\
\hline 1 & 20.396 .000 & 40 & 19 & 509.900 & 1.054 .644 \\
2 & 29.380 .201 & 45 & 43 & 652.893 & 684.299 \\
3 & 29.282 .060 & 60 & 30 & 488.034 & 965.471 \\
4 & 27.932 .476 & 85 & 45 & 328.617 & 619.064 \\
5 & 35.608 .525 & 95 & 29 & 374.827 & 1.230 .930 \\
6 & 17.746 .968 & 31 & 47 & 572.483 & 375.465 \\
7 & 3.856 .018 & 22 & 12 & 175.274 & 334.987 \\
8 & 18.468 .106 & 76 & 31 & 243.001 & 601.124 \\
9 & 16.878 .747 & 47 & 23 & 359.122 & 747.203 \\
10 & 16.274 .266 & 28 & 23 & 581.224 & 711.244 \\
11 & 19.301 .792 & 56 & 25 & 344.675 & 771.345 \\
12 & 1.141 .561 & 16 & 9 & 71.348 & 125.229 \\
13 & 20.754 .150 & 36 & 50 & 576.504 & 416.974 \\
\hline Promedio & 19.770 .836 & 49 & 30 & 405.992 & 664.460 \\
\hline
\end{tabular}

\section{Estructura de costos}

En la Figura 4 se presentan los promedios de los costos de los 13 productores, observándose que los con mayor incidencia en la producción corresponden a la alimentación del ganado (31\%), seguido por la reposición de los animales (22\%), fertilizantes y abonos (11\%) y mano de obra (9\%). Para la alimentación el rango va de un $20 \%$ (agricultor 9) a 46\% (agricultor 5) de los costos totales.

En el caso de los fertilizantes que representan en promedio el $11 \%$ del total de costos, el rango fluctúa entre un 5\% (productor 9) y un 24\% (agricultor 6).Este rango se explica por la diversidad y cantidad de cultivos que siembra cada agricultor, con dosis de fertilizantes que son variables para un mismo cultivo entre agricultores.

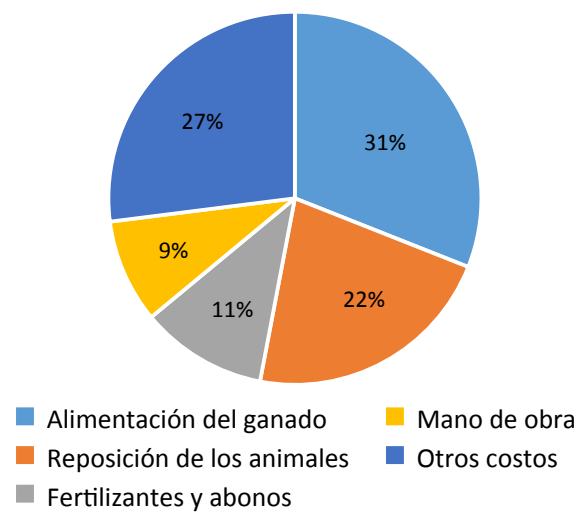

Figura 4. Estructura de los principales costos de las explotaciones $(\%)$.
Fundación Chile (2000), señala que las economías de escalas disminuyen los costos fijos unitarios y trata de encontrar una relación entre las mejores rentabilidades y los costos, señalando que la eficiencia que obtienen algunos productores en alimentación, mano de obra y aspectos reproductivos, hace la diferencia en la eficiencia económica. Al producir heno y ensilaje de alta calidad es recomendable disminuir el uso de concentrando ya que hay una relación en el alza de la rentabilidad al bajar el uso de este tipo de alimento.

Este mismo autor, al analizar los costos en el Centro de Gestión Todoagro Valdivia, encontró que los mayores porcentajes que influyen en los costos del litro de leche pertenece a la alimentación (43\%), depreciación y administración.

\section{Estructura de ingresos}

En la Tabla 2 se presentan los ingresos de los productores expresados en porcentaje, observando que la venta de leche es la más relevante con un promedio de $36 \%$, y un rango de $6 \%$ a $56 \%$ (productores 12 y 11). Este contraste se debe a la diversidad de rubros que mantienen los productores, siendo alguno de ellos el ingreso por venta de papa o en el ítem "otros ingresos" que contempla la venta de granos, aves y leña.

El ingreso por la venta de terneros y terneras presenta un promedio de $16 \%$ con un rango de $4 \%$ a $25 \%$ (productores 3 y 9). Dependiendo del mercado algunos productores optan por criar los terneros y así obtener mejor precio al venderlos 
Tabla 2. Estructura de ingresos de los productores (\%).

\begin{tabular}{|c|c|c|c|c|c|c|c|c|c|c|c|c|c|c|}
\hline Ingresos $(\%)$ & 1 & 2 & 3 & 4 & 5 & 6 & 7 & 8 & 9 & 10 & 11 & 12 & 13 & Prom. \\
\hline Pastoreo & 11 & 12 & 10 & 10 & 11 & 14 & 4 & 8 & 2 & 5 & 13 & 1 & 8 & 8 \\
\hline Venta de vacas desecho leche & 1 & 2 & 0 & 1 & & 2 & 2 & 2 & 4 & 1 & 2 & 1 & 2 & 2 \\
\hline Venta de vaquillas & 6 & 10 & 8 & 19 & 13 & 1 & 2 & 17 & 24 & 10 & 18 & 16 & 9 & 12 \\
\hline Venta de terneros y terneras & 19 & 18 & 4 & 24 & 21 & 8 & 6 & 21 & 25 & 12 & 5 & 20 & 24 & 16 \\
\hline Venta de leche & 49 & 48 & 30 & 42 & 48 & 37 & 14 & 39 & 35 & 19 & 56 & 6 & 41 & 36 \\
\hline Diferencia de inventarios & 2 & & 2 & & 2 & & 1 & 6 & 1 & 1 & 7 & 3 & 2 & 3 \\
\hline Venta de leche a crianza & 2 & 6 & & 2 & 2 & 3 & 1 & 4 & 5 & 4 & 1 & 19 & 3 & 4 \\
\hline Venta de vaquillas preñadas & & & & & & & & & & & & 8 & & 8 \\
\hline Venta de leche a ferias & & 2 & & & & & & & & 31 & & & & 17 \\
\hline Venta de papas & & & 46 & & & & 69 & & & & & 21 & & 45 \\
\hline Subvenciones & 1 & & & & & 6 & & & 2 & 4 & & & 1 & 3 \\
\hline Otras ventas & 10 & 3 & 1 & 1 & 3 & 29 & 1 & 3 & 3 & 12 & 0 & 6 & 10 & 7 \\
\hline
\end{tabular}

como novillos; al igual que las terneras que son criadas para el reemplazo de las vacas y el excedente de vaquillas es vendido, obteniendo este ingreso un promedio del $12 \%$.

En el proceso productivo de leche existen ingresos por venta de vacas de desecho, teniendo un promedio de $2 \%$; la diferencia de inventario presenta un promedio del 3\%, por la reposición de animales, aunque algunos productores están incrementando la masa ganadera.

La cuenta de subvenciones está presente en el ingreso de solo 5 agricultores, obteniendo un promedio general de $3 \%$.

Si bien en la mayoría de los productores el rubro principal es la leche, existen tres que tienen ingreso importante por venta de papas (45\% de los ingresos totales). Este ingreso es, en promedio, el más elevado, aunque no representa la totalidad de los agricultores. Al respecto es importante mencionar que el precio de la papa es fluctuante año a año. Rivas y Tapia (2012), grafican la gran fluctuación de los precios, demostrando una variación cíclica de tres años que va en aumento y luego cae por exceso de oferta.

\section{Beneficio neto total de la explotación}

En la Tabla 3 se presenta el beneficio neto total y por hectárea de los productores, provenientes de la venta de leche, crianza de terneros, vaquillas, novillos; además de los ingresos generados por procesos productivos secundarios, como la producción y comercialización de papa, cerdos, ovinos, etc.

El beneficio neto total más alto lo alcanzó el productor 3 (\$54.627.678), y el más bajo el 8
(\$ 2.703.112), con un promedio de $\$ 14.540 .652$ por predio. Estos resultados indican que, en promedio, los predios generan ingresos suficientes para financiar los costos operacionales. El mayor beneficio neto total generado por el productor 3 , se puede asociar al ingreso por venta de leche, pero además de esta actividad, el agricultor obtiene el $46 \%$ de sus ingresos por venta de papas, produciendo 45,2 toneladas en la temporada. Cabe señalar que no todas sus actividades son rentables, ya que la crianza de vaquillas le genera un margen negativo (-\$4.731.281), por los altos costos en alimentación (concentrado), siendo este déficit financiado por las demás actividades.

El beneficio neto total generado por el agricultor 8 es el más bajo del grupo, en cuya estructura de ingresos (Tabla 3) el 39\% corresponde a la venta

Tabla 3. Beneficio neto total y por hectárea de productores lecheros.

\begin{tabular}{crr}
\hline Agricultor $\left(\mathrm{N}^{\circ}\right)$ & Total $(\$)$ & Total/ha (\$) \\
\hline 1 & 4.915 .350 & 702.193 \\
2 & 12.437 .723 & 170.380 \\
3 & 54.627 .678 & 1.365 .692 \\
4 & 18.579 .860 & 232.249 \\
5 & 14.912 .068 & 298.242 \\
6 & 9.951 .996 & 160.517 \\
7 & 32.335 .263 & 1.701 .856 \\
8 & 2.703 .112 & 56.315 \\
9 & 3.880 .714 & 88.199 \\
10 & 10.100 .921 & 194.249 \\
11 & 6.705 .173 & 209.537 \\
12 & 5.924 .165 & 329.121 \\
13 & 11.954 .452 & 137.408 \\
\hline Promedio & 14.540 .652 & 434.304 \\
\hline
\end{tabular}


de leche, con altos costos en alimentación, y un menor precio de la leche por problemas de calidad higiénica. Este mismo productor, en la actividad crianza de terneros genera un margen negativo (-\$2.739.919), con altos costos en alimentación, además con gastos generales de $-\$ 18.906 .104$, provocando la disminución del beneficio neto total.

Lerdon y Rabanal (1999), en un estudio realizado a 29 agricultores lecheros de la zona de Paillaco, encontró que el beneficio neto de las explotaciones de la categoría C (clasificada por INDAP) era de \$2.293.273 (valor deflactado en función del Índice de Precios al Consumidor y expresado en moneda de mayo de 2013), que se encuentra muy por debajo de los promedios de este estudio, lo que se debe probablemente al uso de nuevas tecnologías o al tipo de agricultor analizado, ya que la categoría $\mathrm{C}$ corresponde a pequeños agricultores.

$\mathrm{Al}$ analizar el beneficio neto por hectárea este fluctúa entre \$ 56.315 (productor 8) y \$ 1.701 .856 (productor 7), con un promedio por agricultor $\$ 434.304$. El productor 7 tiene costos de producción menores al resto. Los ingresos por venta de leche corresponden al $14 \%$ ya que el tamaño de la actividad es de 22 cabezas, obteniendo también el $69 \%$ por la venta de papas. Esta explotación al ser arrendada (24 ha) a \$263.000/ha, su costo es bajo en relación a los ingresos obtenidos.

$\mathrm{Al}$ correlacionar el beneficio neto/ha con la edad de los agricultores (Tabla 6), se observa que esta es negativa, es decir, mientras aumenta el beneficio neto/ha la edad disminuye $(r=-0,49)$. Llama la atención que una situación similar se da al correlacionar esta variable con el nivel de escolaridad $(r=-0,33)$.

\section{Antecedentes financieros y económicos}

Se analizan los principales indicadores relacionados con la solvencia, liquidez y rentabilidad, pudiéndose observar importantes diferencias entre agricultores, dependiendo del rubro a que se dedican y si la explotación que trabajan es propia o arrendada.

\section{Indicadores de solvencia}

Para analizar las relaciones entre los capitales propios y ajenos se utilizaron tres indicadores: tasa de solvencia, tasa de autonomía financiera y grado de endeudamiento, cuyos resultados se presentan en la Tabla 4.
La tasa de solvencia mide la relación entre los capitales propios y el pasivo total. Un monto elevado de capitales propios, asegura una cierta independencia o autonomía de la empresa frente a los acreedores, permitiendo acceder a créditos en mejores condiciones, cuando estos son necesarios para asegurar el funcionamiento y desarrollo de la empresa (Lerdon, 2011). El mismo autor recomienda que esta tasa sea superior al $60 \%$, lo que se traduce en que los capitales propios financien al menos el $60 \%$ del activo total.

En promedio, el $87 \%$ de los activos totales de los 13 productores, están siendo financiados con capitales propios y el $15 \%$ restante vía crédito. Prácticamente la totalidad de los agricultores tiene una buena tasa de solvencia, que supera el $60 \%$, excepto los agricultores 6 y 7 los que alcanzan un $59 \%$, índice similar al de referencia.

Los agricultores 8 y 12 llegan al máximo 100\%, no presentando ningún tipo de deuda.

Lerdon et al. (2008), determinaron que la tasa de solvencia en 16 predios campesinos lecheros, era en promedio de $90 \%$, similar a los predios analizados.

La tasa de autonomía financiera mide la relación existente entre las deudas a mediano largo plazo con el capital propio. Según Lerdon (2011), una empresa rentable no debería endeudarse a mediano o largo plazo en un monto superior a su capital propio, y recomienda que este indicador sea superior al $100 \%$.

Se observa en la Tabla 4 que el promedio de este indicador es de $1.671 \%$, con un rango que va desde $80 \%$ para el agricultor 7 (único que está por debajo de lo recomendado), hasta $6.169 \%$ correspondiente al agricultor 2, obteniendo los indicadores más altos los productores de carne.

Lerdon y Rautenberg (2001), en seis predios lecheros de la comuna de Paillaco encontraron que la tasa de autonomía financiera presentaba un rango entre $416 \%$ y $4.483 \%$, el que presenta un mayor grado de solvencia al observado en este estudio.

El grado de endeudamiento mide la relación entre el pasivo exigible y el pasivo total, expresada como porcentaje (Lerdon, 2011). Como se observa en la Tabla 4, y en concordancia con el análisis anterior, en la mayoría de los casos el grado de endeudamiento es bajo, excepto en los agricultores 6 y 7 , ambos con un $41 \%$, encontrándose este indicador en el máximo recomendado, superando el $40 \%$; el promedio obtenido es de un 11\%. En efecto, Lerdon (2011), señala que el grado de endeudamiento no debería sobrepasar el $40 \%$, para que los capitales propios 
Tabla 4. Indicadores de solvencia.

\begin{tabular}{cccc}
\hline $\begin{array}{c}\text { Agricultores productores de leche } \\
\left(\mathrm{N}^{\circ}\right)\end{array}$ & $\begin{array}{c}\text { Tasa de solvencia } \\
(\%)\end{array}$ & $\begin{array}{c}\text { Tasa de autonomía financiera } \\
(\%)\end{array}$ & $\begin{array}{c}\text { Grado de endeudamiento } \\
(\%)\end{array}$ \\
\hline 1 & 92 & 1.163 & 8 \\
2 & 97 & 6.169 & 3 \\
3 & 99 & & 1 \\
4 & 94 & 1.603 & 6 \\
5 & 68 & 234 & 32 \\
6 & 59 & 190 & 41 \\
7 & 59 & 50 & 19 \\
8 & 100 & 553 & 2 \\
9 & 81 & 1.245 & 8 \\
10 & 98 & & 4 \\
11 & 92 & 3.802 & 15 \\
\hline
\end{tabular}

financien como mínimo el $60 \%$ de los activos de la empresa. Lerdon y Rautenberg (2001), encontraron que el grado de endeudamiento presentaba un rango de $1 \%$ a $17 \%$, cifras inferiores a las observadas en este estudio.

Respecto de la correlación entre el grado de endeudamiento y la edad, y entre este mismo indicador y el nivel de escolaridad, de acuerdo a las cifras que aparecen en la Tabla 6 , se puede señalar que en el primer caso esta no es clara $(r=-0,12)$. Para la segunda correlación se tiene que mientras aumenta el grado de endeudamiento, disminuye la edad $(r=-0,51)$.

\section{Indicadores de liquidez}

Estos índices permiten conocer la capacidad de los agricultores para cubrir sus deudas a corto plazo, considerándose en este estudio lo siguiente: capital de operación, necesidad en capital de operación y situación de liquidez, cuyos resultados se presentan en la Tabla 5. El capital de operación es la diferencia entre el activo circulante y el pasivo circulante, e indica si el primero permite financiar deudas a corto plazo de la empresa (Lerdon, 2011).

El promedio del capital de operación fue de \$ 7.376.335 con un rango de $\$ 136.620$ a \$ 31.907 .000 (agricultores 6 y 5 , respectivamente).

La necesidad en capital de operación está determinada por el stock total más la diferencia entre los créditos a clientes y los créditos otorgados por los proveedores (Lerdon, 2011).
Los agricultores, en promedio, necesitan $\$ 7.648 .194$ para su normal funcionamiento, encontrándose un rango de $\$ 136.620$ a \$31.907.000 (productor 6 y 5, respectivamente), siendo el último el que más dinero necesita para financiar la operación de su empresa.

Finalmente, la liquidez corresponde a la diferencia entre el capital de operación y las necesidades en capital de operación, observándose que 7 de los 13 agricultores presentan cifras negativas, con un rango que va desde $-\$ 6.160 .035$ (agricultor 6) a $\$ 9.000 .000$ (agricultor 5). El promedio fue de -\$271.859, lo que indica que el capital de operación no cubre las necesidades en capital de operación, con un déficit promedio de $\$ 271.859$.

\section{Indicadores de rentabilidad}

En la Figura 5 se presenta la rentabilidad financiera y económica de los predios. La rentabilidad financiera o de los capitales propios indica la tasa de interés que reditúan los fondos propios invertidos por los agricultores, observándose que este indicador es positivo para los 13 agricultores lecheros, con un promedio de $17 \%$ y valores que fluctúan entre $1 \%$ a $127 \%$ para los productores 8 y 7 . Este último presenta una rentabilidad muy superior a lo normal en la agricultura tradicional, lo que se debe, por una parte, a los altos ingresos que percibe por actividades secundarias (venta de papas) y, por otra, a que su explotación la realiza en un predio arrendado, condición que mejora la rentabilidad por la baja inversión. 
Tabla 5. Indicadores de liquidez.

\begin{tabular}{|c|c|c|c|}
\hline $\begin{array}{l}\text { Agricultores productores de leche } \\
\qquad\left(\mathrm{N}^{\circ}\right)\end{array}$ & $\begin{array}{l}\text { Capital de operación } \\
\text { (\$) }\end{array}$ & $\begin{array}{c}\text { Necesidad en capital de operación } \\
(\$)\end{array}$ & $\begin{array}{l}\text { Liquidez } \\
\quad(\$)\end{array}$ \\
\hline 1 & 8.630 .000 & 8.520 .000 & 110.000 \\
\hline 2 & 3.950 .960 & 6.010 .960 & -2.060 .000 \\
\hline 3 & 10.700 .000 & 5.100 .000 & 5.600 .000 \\
\hline 4 & 9.437 .410 & 8.953 .410 & 484.000 \\
\hline 5 & 31.907 .000 & 22.907 .000 & 9.000 .000 \\
\hline 6 & 136.620 & 6.296 .655 & -6.160 .035 \\
\hline 7 & 4.900 .000 & 5.400 .000 & -500.000 \\
\hline 8 & 9.870 .800 & 9.600 .800 & 270.000 \\
\hline 9 & 941.863 & 4.800 .000 & -3.858 .137 \\
\hline 10 & 350.000 & 3.020 .000 & -2.670 .000 \\
\hline 11 & 3.050 .000 & 3.400 .000 & -350.000 \\
\hline 12 & 2.192 .700 & 1.792 .700 & 400.000 \\
\hline 13 & 9.825 .000 & 13.625 .000 & -3.800 .000 \\
\hline Promedio & 7.376 .335 & 7.648 .194 & -271.859 \\
\hline
\end{tabular}

Tabla 6. Resultados del análisis de correlaciones (Pearson).

\begin{tabular}{lrc}
\hline & Edad & Nivel de escolaridad \\
\hline Rentabilidad capital propio & $-0,29$ & $-0,12$ \\
Rentabilidad capital total & $-0,32$ & $-0,09$ \\
Margen directo/cab & 0,34 & $-0,18$ \\
Margen directo/ha & 0,22 & $-0,26$ \\
Beneficio neto/ha & $-0,49$ & $-0,33$ \\
Grado de endeudamiento & $-0,12$ & $-0,51$ \\
\hline
\end{tabular}

Lerdon et al. (2008) encontraron un promedio de rentabilidad de los capitales propios de 2,1\%, valor inferior al observado en este estudio. Por su parte, Lerdon y Rautenberg (2001), determinaron rentabilidades de los capitales propios para el rubro lechería que fluctuaban entre $-0,2 \%$ y $9 \%$, los que están dentro del rango de los encontrados en este estudio.

Al observar el índice de correlación entre la rentabilidad del capital propio y la edad de los agricultores (Tabla 6), se observa que, mientras esta aumenta, la edad disminuye $(r=-0,29)$. En el caso de la correlación de este indicador y el nivel de escolaridad esta no es clara $(r=-0,12)$.

La rentabilidad económica o de los capitales totales analiza la capacidad efectiva de la explotación para remunerar todos los capitales puestos a su disposición, ya sean propios o de terceros (Lerdon, 2011). En la Figura 5 se observa que los productores obtienen rentabilidades que fluctúan entre $1 \%$ a $79 \%$ (agricultores 8 y 7 ), con un promedio de $13 \%$.
Fundación Chile (2002), señala que para mejorar la rentabilidad es necesario mejorar la gestión productiva, mediante la planificación anual y semanal. Al igual que el uso de la gestión financiera, usando contabilidad y el uso de un orden meticuloso. Este mismo autor señala que los agricultores con mejor desempeño económico se caracterizan por usar un mejor control de gestión ya que utilizan los indicadores por medio de las temporadas llevando las actividades sistemáticamente.

En cuanto a la correlación entre la rentabilidad del capital total y la edad de los agricultores (Tabla 6), se tiene que mientras el primer indicador aumenta, la edad disminuye $(r=-0,32)$. La correlación con el nivel de escolaridad no es clara $(r=-0,09)$.

\section{Conclusiones}

- La edad promedio de los agricultores fue de 46 años, encontrándose por debajo de lo observado por otros estudios similares. Condición favorable para el grupo, ya que con menor edad existiría una mayor apertura al uso de nuevas tecnologías.

- El $62 \%$ tenía estudios técnicos, de los que algunos son agrarios, lo que al momento de la toma de decisiones juegan un rol importante. Si se compara el beneficio neto con la escolaridad, los agricultores con estudios agrarios obtienen cifras mayores.

- El número de mujeres jefes de la explotación es mayor que el de los hombres. Si se compara en promedio la rentabilidad de los capitales propios 


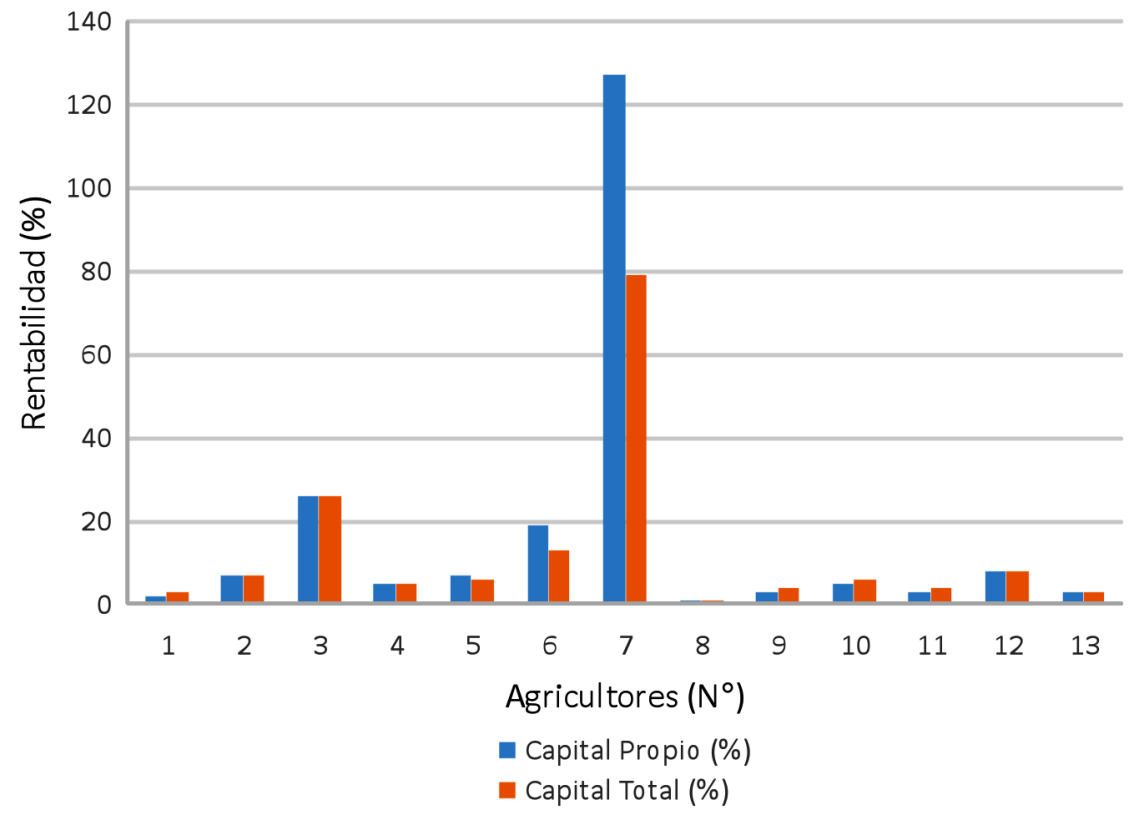

Figura 5. Rentabilidad de los capitales propios y totales.

y totales, los hombres presentan indicadores mayores que las mujeres.

- Los costos directos más importantes están representados por la alimentación, reposición, fertilizantes y abonos, y son similares a otros estudios realizados en la zona de análisis.

- De los ingresos generados el $64 \%$ proviene de la venta de leche, venta de vaquillas, terneros y terneras. Existen agricultores que producen papas, lo que representa un porcentaje importante de sus ingresos.

- La liquidez es negativa en 7 de los 13 predios analizados, debido a las altas necesidades en capital de operación, existiendo deudas a corto plazo, que se traducen muchas veces en un círculo vicioso, pues para cancelar estas deudas se recurre a sobregiros o a nuevas deudas. También influye en este indicador el mayor nivel de stock existente en algunos predios.
- La tasa de solvencia en todos los predios es mayor al $60 \%$ y el grado de endeudamiento total es bajo.

- La rentabilidad de los capitales propios es, en promedio, de $17 \%$, superior a lo encontrado por otros autores. La rentabilidad del capital total (13\%) fue levemente inferior a la anterior, trabajando la mayoría de los agricultores con recursos propios.

- Las explotaciones más grandes (hectáreas o en número de cabezas), no obtuvieron las mejores rentabilidades. Asimismo, los predios arrendados tienen rentabilidades mayores, ya que esta forma de producir si bien genera costo de arriendo, disminuye significativamente el nivel de inversión.

- La mayor correlación positiva se registró entre la edad y el margen directo por cabeza de ganado.

- La mayor correlación negativa se registró entre grado de endeudamiento y el nivel de escolaridad.

\section{Literatura Citada}

Apey, A.; Tapia, B.; Ramírez, J.; Muñoz, A.; Guevara, G. y Muñoz, L. 2005. Agricultura Chilena: características sociales de los productores según tipología, sexo y localización geográfica. Chile. Oficina de Estudios y Políticas Agrarias (ODEPA), 109 p.

\section{Becerra, L.}

2002. El abc de los GTT. Instituto de Investigaciones Agropecuarias (INIA). Estación Experimental Quilamapu. Chillán, Chile. Boletín INIA N ${ }^{\circ} 77$. Ministerio de Agricultura, pp. 3-23. 
Díaz, C.

2005. Mujeres rurales en Chile. Informe elaborado para el Servicio Nacional de la Mujer (SERNAM) y para la Organización de las Naciones Unidas para la Agricultura y la Alimentación (FAO). Santiago. Disponible en: ftp://ftp. fao.org/docrep/fao/010/ai019s/ai019s00.pdf. Consultado: $5 /$ jun/2013.

Domínguez, J.

2000. Una visión prospectiva para la agricultura chilena. En. Furche, C. (ed.). Agricultura Chilena del 2010. Tres visiones sociopolíticas. Ministerio de Agricultura, Santiago, pp. 91-128.

Echenique, $\mathrm{J}$.

2004. Tendencias y papel de la tecnología en la agricultura familiar del cono sur. Instituto Interamericano de Cooperación para la Agricultura (IICA). Montevideo, pp. 9-12.

Fundación Chile.

2000. Economía y gestión de la producción lechera. La Fundación, Santiago, 65 p.

Gordillo, G. y Jiménez, F.

2004. El nuevo eje de la seguridad alimentaria. (On line): IAI-IHDP. 2004. Global Environmental Change Institute on Globalization and Food Systems. Disponible en: http://www. bvsde.paho.org/bvsacd/cd67/eje.pdf. Consultado: 10/ jun/2012.

Hofer, A.

2011. Informe silvoagropecuario región de la Araucaníaenero/febrero. Secretaría Regional Ministerial de Agricultura, Ministerio de Agricultura. Temuco, 6 p.

Instituto Interamericano de Cooperación para la Agricultura (IICA). 2004. Situación y perspectivas de la agricultura y de la vida rural en las Américas. IICA. San José, Costa Rica, 68 p.

Instituto Nacional de Estadística (INE).

2007. Cambios estructurales en la agricultura chilena-análisis intercensal 1976-1997-2007. Disponible en: http://www. ine.cl/canales/chile_estadistico/censos_agropecuarios/ censos_agropecuarios.php. Consultado: 25/ jun/2013.

Lerdon, J.

2012a. Sistema de Contabilidad de Gestión Agrícola (CONGA). Guía del usuario, $40 \mathrm{p}$.

Lerdon, J.

2012b. Capacitación a agricultores en uso de tecnología y gestión para aumentar la competitividad del rubro. Disponible en: http://www.agrarias.uach.cl/noticia.php?codigo=13402 Consultado: 10/abr/2012.

Lerdon, J.

2011. Análisis financiero de empresas agrícolas. Universidad Austral de Chile, Facultad de Ciencias Agrarias, Instituto de Economía Agraria, Valdivia, 101 p.

Lerdon, J.; Rautenberg, R.

2001. Estimación de eficiencia de producción y análisis económico de seis predios lecheros de la comuna de Panguipulli. Estudio de casos. Agro Sur, 29 (2): 149-163.

Lerdon, J.; Báez, A. y Azócar, G.

2008. Relación entre variables sociales, productivas y económicas en 16 predios campesinos lecheros de la provincia de Valdivia, Chile. Archivos de Medicina Veterinaria, 40 (2): 179-185.

Lerdon, J.; Rabanal, I.

1999. Análisis económico y financiero de 29 predios campesinos lecheros de Paillaco. Agro Sur, 27 (2): 145-166.

Navarro, $\mathrm{H}$.

2006. Antecedentes de la producción de leche en el sur de Chile. En: Navarro, H.; Siebald, E.; Celis, S. Manual de producción de leche para pequeños y medianos productores. Boletín 148. INIA-Centro Regional de Investigación Remehue. Santiago, Chile, pp. 2-11.

Organización para la Cooperación y el Desarrollo Económico (OCDE)

2009. Estudios territoriales de la ODCE. (Chile). Ministerio del Interior. Santiago, Chile, $243 \mathrm{p}$.

Pulido, R.; Parga, J.; Lanuza, F. y Balocchi, O.

2011. La pradera como alimento. En: Pulido, R.; Parga, J.; Lanuza, F. y Balocchi, O. Suplementación de vacas lecheras a pastoreo. Consorcio Lechero. Osorno, Chile, pp. 11-20. Disponible en: http://www.consorciolechero.cl/chile/docs/ suplementacion-de-vacas-lecheras-a-pastoreos-v2.pdf. Ramírez, E.

2002. El Mercado de Arriendo de Tierras en Chile (estudio de caso). Organización de las Naciones Unidas para la Agricultura (FAO). Centro Latinoamericano para el Desarrollo Local, 28 p. Consultado: 04/abr/2016

Rivas, T. y Tapia, B.

2012. Estructura de mercado y determinantes del precio de la papa para consumo fresco. Oficina de Estudios y Políticas Agrarias (ODEPA). Disponible en: http://www. odepa.gob.cl//odepaweb/publicaciones/doc/8636.pdf; j sessionid=3319CE16781FEAFA1B1FB08E15911F4D Consultado: 15/ jun/ 2013.

Sandoval, C. y Gómez, J. 1999. Los Centros de Gestión Empresarial en Chile. Instituto de Desarrollo Agropecuario. Santiago, 148 p.

Smith, R.; Moreira, V. y Latrille, L.

2002. Caracterización de sistemas productivos lecheros en la X Región de Chile mediante análisis multivariable. Agricultura Técnica, Chile, 62 (3): 375-395.

Villalobos, $\mathrm{P}$.

2002. Instituto de Desarrollo Agropecuario (INDAP). En: IICA. Buenas prácticas agrícolas: eje estratégico de nuestra competitividad futura. IICA - Secretaria de Agricultura. Santiago, Chile, pp. 115-125. 